\title{
Progress in the study of pulsating subdwarf B stars
}

\author{
M. D Reed \\ Department of Physics, Astronomy, \& Materials Science, \\ Missouri State University, $901 \mathrm{~S}$ National Ave, Springfield, MO, 65802 USA \\ email: mikereed@missouristate.edu
}

\begin{abstract}
During Kepler's main mission, nearly 20 pulsating subdwarf B (sdB: extreme horizontal branch stars) were discovered. Many of these stars were observed for three years, accumulating over 1.5 million observations. Only through these extended observations have we been able to identify pulsation modes, applying constraints for structure models. Discoveries include nearly-evenly-spaced asymptotic period overtones which represent the interior structure and rotationally-induced frequency multiplets from which we have learned that rotation periods are long, even when in short-period binaries. This paper reviews progress on observational constraints and highlights some of our discoveries including radially differential rotation, conflicting stratification indicators and mode lifetimes.
\end{abstract}

Keywords. Kepler, asteroseismology

\section{Introduction}

Subdwarf B (sdB) stars are hot horizontal branch stars with $\sim 20000<T_{\text {eff }}<$ $40,000 \mathrm{~K}$. In clusters they are referred to as extreme horizontal branch or blue hook stars (Moheler et al. 1998) with masses near $0.5 M_{\odot}$ and thin $\left(<10^{-2} M_{\odot}\right)$ hydrogen shells (Heber 1984, Saffer et al. 1994). These stars have somehow shed their outer layers near the tip of the red giant branch and so represent the exposed cores of most horizontal branch stars. Pulsators were first observed in 1995 and now are divided into two classes: V361 Hya stars are short-period, $p$-mode pulsators with periods of minutes and amplitudes typically near $1 \%$ and V1093 Her stars which are longer-period, $g$-mode pulsators with periods of an hour or two and usually lower amplitudes. The V1093 Her stars tend to be cooler than the V361 Hya stars, but there is overlap and hybrid pulsators. For a review of the stellar properties, see Heber (2009) and Østensen (2010) for a review of the pulsation properties.

The Kepler spacecraft's primary mission was to discover and characterize extrasolar planet transits (Jenkins et al. 2010), but as it observed stars continuously for at least three years, Kepler was incredibly useful for asteroseismology. Over a dozen papers have been published applying asteroseismological tools to Kepler data on sdB stars. The review in this proceedings by Charpinet et al. primarily covers modeling efforts and applications to observations and this paper will focus on observational tools and constraints which have come from Kepler data.

\section{Asteroseismic tools}

Standard observational tools to correlate pulsation modes with quantized indices $n$ (or $k$ ), $\ell$, and $m$ include asymptotic period spacing and frequency multiplets. In the asymptotic limit $(n \gg \ell)$, stellar pulsations in an idealized homogeneous star appear as 


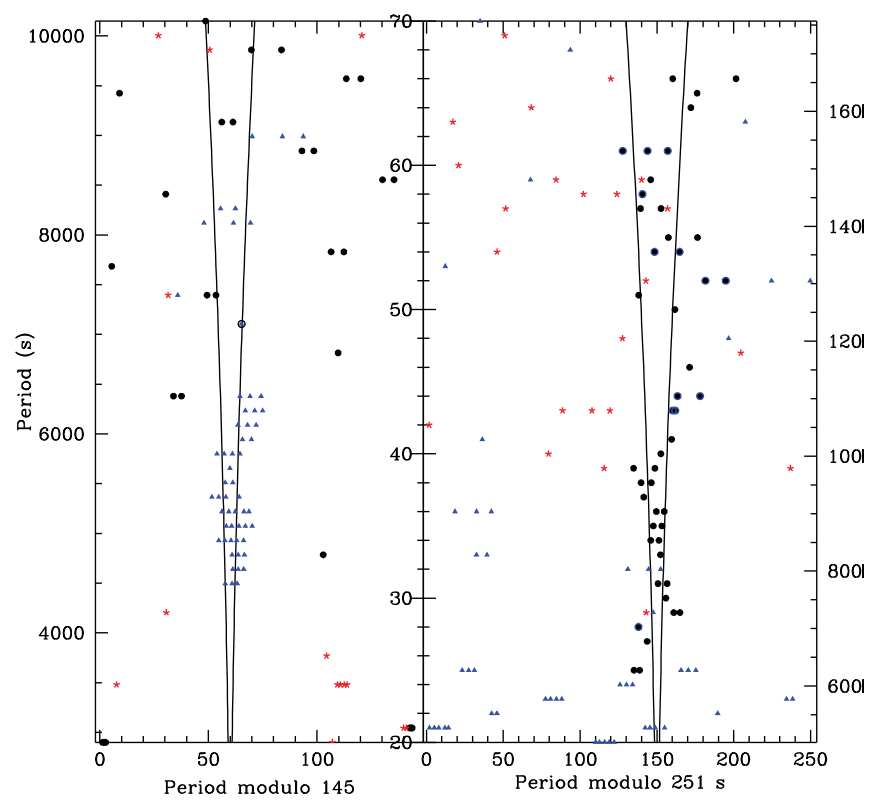

Figure 1. Echelle diagrams appropriate for $\ell=1$ and 2 modes.

standard harmonic oscillators, to which $g$-mode periods follow the relationship

$$
\Pi_{\ell, n}=\frac{\Pi_{o}}{\sqrt{\ell(\ell+1)}} n+\epsilon
$$

where $\Pi_{o}$ and $\epsilon$ are constants with units of time (see Aerts, Christensen-Dalsgaard, \& Kurtz 2010; Unno et al. 1979; among others).

Pulsation modes can be identified if they follow this equation, especially as they relate between various degrees. A useful visualization tool is the Echelle diagram which folds pulsation periods over a period, usually indicated by a Kolomogoroz-Smirnov test. Figure 1 shows such a plot for KIC 10670103 for $20 \leqslant n \leqslant 70$. For this star, the regions between $n \sim 30$ and 45 are nearly pure for each sequence. Table 1 summarizes the period spacings found for each of the Kepler-observed sdB pulsators.

Frequency multiplets are typically caused by rotation, which removes the azimuthal degeneracy (Ledoux 1951). Frequencies are separated by azimuthal order $(-\ell$ to $\ell$ ) as

$$
\Delta \nu=\Delta m \Omega\left(1-C_{n, \ell}\right)
$$

where $\Delta \nu$ represents the shift from the degenerate or $m=0$ mode frequency, $\Omega$ is the frequency of stellar rotation, and $C_{n, \ell}$ is the Ledoux constant. The Ledoux constant is small for $p$-modes, but for higher-order $g$-modes it depends on the degree, $\ell$, according to

$$
C_{n, \ell} \approx \frac{1}{\ell(\ell+1)}
$$

As the $m$ index has $2 \ell+1$ possible values, triplets usually indicate $\ell=1$ modes, quintuplets $\ell=2$ modes, and so on. Of course not all members of a multiplet will necessarily be excited to observable amplitudes, but the value of the Ledoux constant can be used as a second part to this tool. If Equation 2.3 is true, then all multiplet members for any degree will have the same Ledoux constant and will be separated by equal amounts in frequency. Once complete multiplets are detected, this can be used to 
Table 1. Period spacings determined from linear least-squares fits. Column 1 provides the KIC number, Columns 2 and 3 are the $\ell=1$ and 2 period spacings (errors in parentheses), columns 4,5 , and 6 provide the total number of periods, and the numbers assigned as $\ell=1$ and 2 . Column 7 is the corresponding reference.

\begin{tabular}{|l|c|c|c|c|l|l|}
\hline KIC & $\begin{array}{c}\Delta \Pi_{1} \\
{[\mathbf{d}]}\end{array}$ & $\begin{array}{c}\Delta \Pi_{2} \\
{[\mathbf{d}]}\end{array}$ & $N$ & $N_{1}$ & $N_{2}$ & Reference \\
\hline 2697388 & $240.07(0.27)$ & $138.54(0.16)$ & 36 & 16 & 13 & Reed et al. $(2011)$ \\
2991403 & $268.52(0.74)$ & $153.84(1.19)$ & 16 & 7 & 4 & Reed et al. $(2011)$ \\
3527751 & $266.4(0.2)$ & $153.2(0.2)$ & 251 & 35 & 95 & Foster et al. $(2015)$ \\
5807616 & $242.12(0.62)$ & $139.13(0.38)$ & 22 & 11 & 6 & Reed et al. $(2011)$ \\
7664467 & 263 & 151 & 61 & 24 & 8 & Baran et al. $(2015 \mathrm{~b})$ \\
7668647 & 248 & 142.2 & 132 & 28 & 45 & Telting et al. (2014) \\
8302197 & $258.61(62)$ & 149 & 30 & 14 & 10 & Baran et al. (2015a) \\
9472174 & $255.63(0.30)$ & $147.70(0.69)$ & 20 & 8 & 8 & Reed et al. $(2011)$ \\
10001893 & $268.6(8)$ & 155 & 156 & 51 & 41 & Reed et al. (in prep.) \\
10553698 & 263.15 & 151.00 & 162 & 81 & 80 & Østensen et al. (2014) \\
10670103 & $251.6(2)$ & $145.0(1)$ & 278 & 67 & 86 & Reed et al. (2014) \\
11179657 & $259.6(14)$ & - & 43 & 29 & 5 & Baran \& Winans (2012) \\
11558725 & $248.87(18)$ & $143.44(08)$ & 166 & 57 & 76 & Telting et al. (2012) \\
\hline
\end{tabular}

Notes:

${ }^{1}$ Using just the $\ell=1$ sequence with five consecutive overtones. ${ }^{2}$ Leaving three deviant $\ell=2$ matches as unassigned.

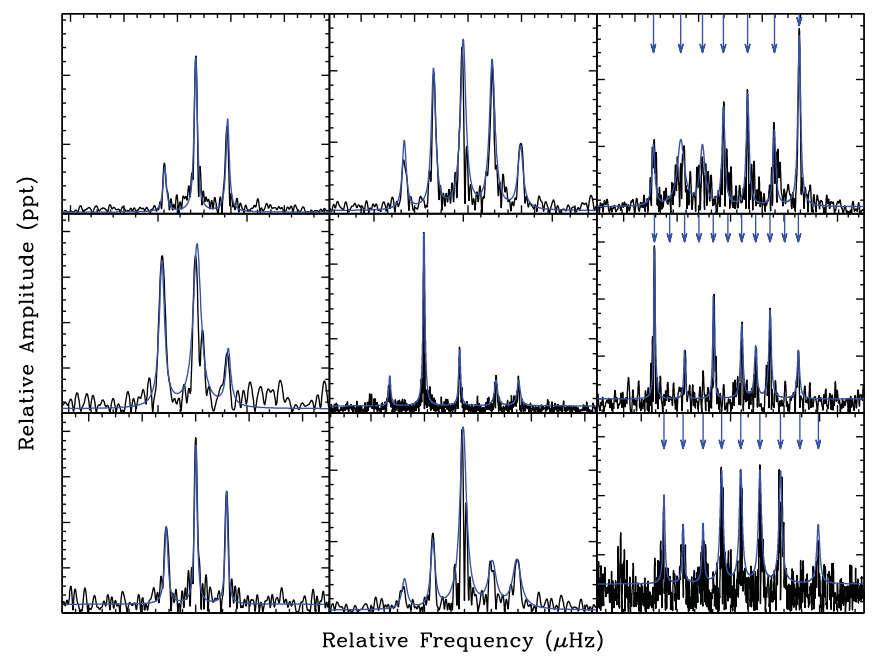

Figure 2. Frequency multiplets. $\ell=1$ multiplets in the left panels, $\ell=2$ in the middle panels and $\ell \geqslant 3$ in the right panels. Arrows are drawn in the right panels to indicate frequency locations, including missing multiplet members.

assign identifications to incomplete ones. Figure 2 shows samples of multiplets detected in Kepler data.

Frequency multiplets also include information about stellar rotation. The frequency multiplets are split in relation to their degree and the rotational frequency. So multiplets provide the stellar rotation period. Table 2 lists frequency multiplets and stellar rotation periods derived from those multiplets.

The data sets obtained by Kepler are unprecedented in the pulsation community and tools which were previously not applied to sdB pulsators became useful. The previously "standard" procedure of prewhitening lightcurves is not useful when the amplitudes, and sometimes the frequencies are varying significantly over the three years of observations. Instead, we fitted Lorentzians and used the Lorentzian width to provide an indicator of amplitude/frequency variation. This is a well-used tool for solar-like oscillations, where 
Table 2. Known rotation periods derived from multiplets.

\begin{tabular}{|l|c|c|c|c|l|l|}
\hline KIC & $\begin{array}{c}P_{\text {rotation }} \\
{[\mathbf{s}]}\end{array}$ & $\begin{array}{c}P_{\text {binary }} \\
{[\mathbf{s}]}\end{array}$ & $N_{1}$ & $N_{2}$ & $N_{>2}$ & Reference \\
\hline 2697388 & $88(8)$ & - & 36 & 16 & 13 & Baran (2012) \\
2991403 & 10.46 & $153.84(1.19)$ & 16 & 7 & 4 & Baran et al. $(2012)$ \\
3527751 & $15.3(7) / 42.6(3.4)$ & - & 9 & 22 & 4 & Foster et al. $(2015$ \\
5807616 & 39.2 & - & 2 & - & - & Charpinet et al. $(2011)$ \\
7664467 & $35.1(6)$ & 1.56 & 6 & 3 & 2 & Baran et al. $(2015)$ \\
7668647 & $44.5(3.5)$ & $14.174(4)$ & 8 & 14 & 3 & Telting et al. $(2014)$ \\
8302197 & 2 years & - & - & - & - & Baran et al. $(2015)$ \\
10001893 & $104(15)$ & - & 10 & 5 & - & Reed et al. $($ in prep.) \\
10553698 & 42.9 & 3.387 & 21 & 15 & - & Østensen et al. $(2014)$ \\
10670103 & $88(8)$ & - & 21 & 23 & - & Reed et al. $(2014)$ \\
11179657 & 7.2 & 0.39 & 5 & 1 & - & Pablo et al. $(2012)$ \\
11558725 & 45 & 10.05 & 21 & 15 & - & Telting et al. $(2012$ \\
\hline
\end{tabular}
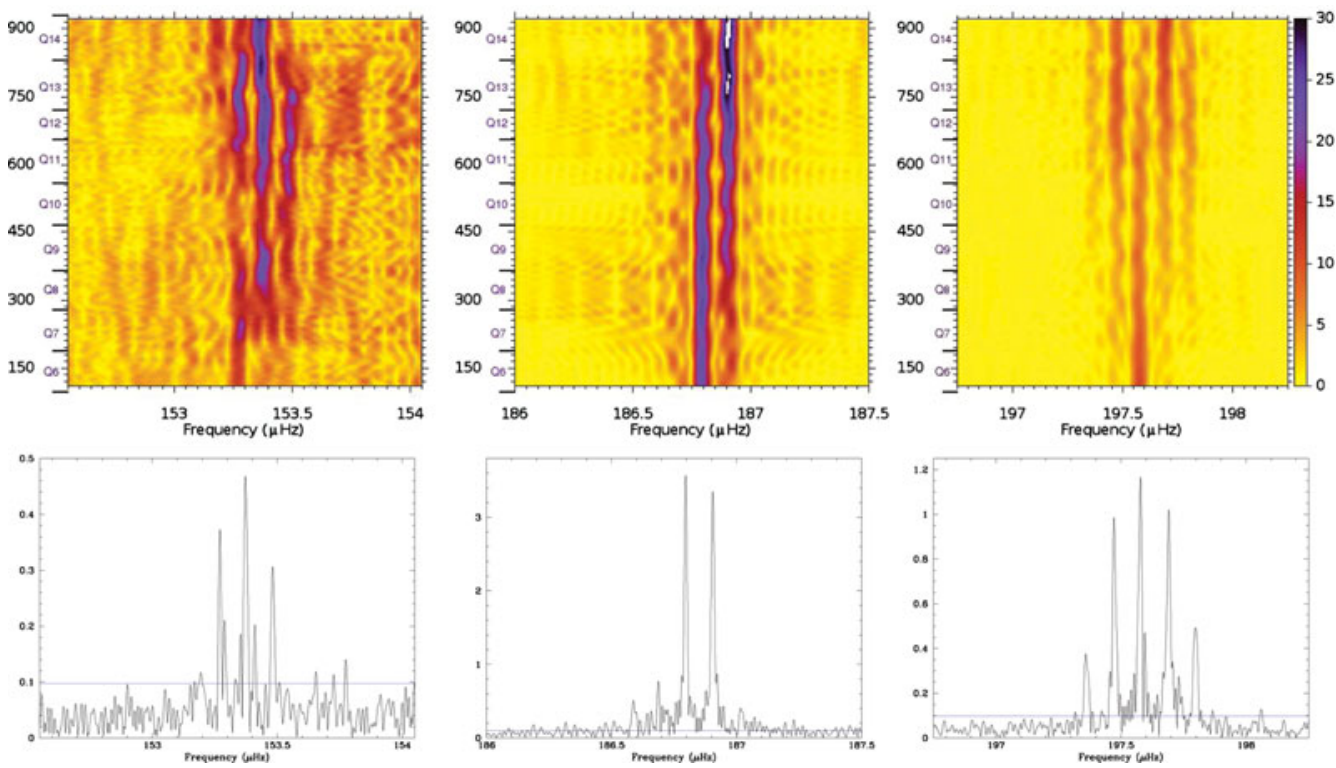

Figure 3. Sliding Fourier transforms (sFTs; top panels) shown with FTs of the combined data set (bottom panels). Each line of the sFTs is a separate FT which spans 220 days and the ordinate indicates the mid-point of the data. in the sFTs. Color indicates amplitude in standard deviations (calculated as the average value of all constituent FTs) with a scale bar on the right.

the widths correspond to stochastic time-scales of interest. At this point however, it seems unlikely that pulsating sdB stars are truly stochastic oscillators, though exceptions may exist, and so we are using Lorentzian fitting as a tool which is solely phenomenological. The blue lines in Fig. 2 indicate the Lorentzian fits for those peaks.

Another device which has proven useful are sliding Fourier transforms (Jacobsen \& Lyons 2003; hereafter called sFTs). These allow FTs to be resolved both in frequency and time over the course of the observations. Individual FTs are produced for a lightcurve of sufficient duration to resolve multiplets and then the data are stepped by some small amount and another FT is produced of equal duration. In this manner, the FTs are stepped in time and stacked. Sample sFTs are shown in Fig. 3 where the abscissa is frequency, the ordinate is time, and the color indicates the amplitude of each FT in sigma. 
Using sFTs, we can examine how the pulsations evolve over the duration of observations. For several stars, interesting phenomena occur. For KIC 10670103 (the examples in Fig. 3), some multiplets appear as singlets during the first months of observations, but then amplitudes increase for the remaining multiplet members to complete the multiplets. The most obvious explanation would be that the pulsation axis of these pulsations have evolved with time. However, only about five modes show this effect, with the others have more random amplitude variations which are not in phase. As such, it is unlikely the pulsation axis is changing. What would have occurred if observed longer? We also have one example where the pulsation frequencies of the $\ell \geqslant 2$ multiplets narrow over the duration of the Kepler observations. At the rate of narrowing, the multiplets would become singlets in about a decade. This seems very unlikely with a more likely explanation some heretofore unseen long-duration periodic trend. However, there is no explanation for what is occurring and we are again left to wonder what would have occurred if Kepler could observe this field for another three years.

Prior to Kepler observations, structure models predicted that sdB stars had a dense core surrounded by an extremely thin atmosphere of roughly equal radius (e.g. Charpinet et al. 2002). Such stratification would lead to extreme mode trapping which would prohibit even period spacings. While we $d o$ observe nearly even period spacings, a measure of even modest mode trapping would be useful for modelling where the atmosphere transitions to the core. A method to search for trapped modes is to use a reduced period plot. Periods are converted to degree-independent values, or reduced periods Pi, by multiplying the period by $\sqrt{\ell(\ell+1)}$. Ostensen et al. (2014) used this method to discover trapped modes in KIC 10553698 which has an uninterrupted series of $21 \ell=1$ and 17 $\ell=2$ overtones. Their Fig. 10 is shown in the left panel of Fig. 4 and shows a series of three trapped modes. In contrast, the right panel of Fig. 4 shows the reduced periods of KIC 10670103, which is another star with long sequences of overtones. The red dashed vertical lines indicate the nearly pure $\ell=2$ sequence described in Reed et al. (2014) which has no trapped modes.

\section{Implications}

Now that we can effectively apply our seismology tools to pulsating sdB stars we can extract some physical insight beyond merely identifying pulsations modes.

The even period spacings provide a challenge to pulsation models, which predicted strong mode trapping breaking up evenly-spaced periods (e.g. Charpinet et al. 2002). A possible solution has been sought in the form of increased diffusion ( $\mathrm{Hu}$ et al. 2009, Miller Bertolami et al. 2012), but so far no models have been able to produce period spacings as consistent as observed (see Reed et al. 2011 for a full discussion of sdB $g$-mode period spacings). Even when mode trapping does occur, it does not appear in a similar fashion to models; compare Fig. 10 of Østensen et al. (2014) (the left panel of Fig 4 above) which has trapped modes with Fig 3 of Reed et al. (2014) which shows model overtone spacings. Observed trapped modes appear as individual deviants within a smooth overtone sequence whereas the models (circa 2010) produce no smooth sequence at all. While challenging, there are now several groups, working with MESA (Paxton et al. 2011) and updated Montreal (Van Grootel et al. 2013) models and so we anticipate progress in the near future. Recent work by Charpinet et al. (2014) indicates that the shape of the overtone sequence is related to the $\mathrm{H} / \mathrm{He}$ boundary and evolves as the boundary does. As such, it could be hoped that, once models can reproduce the observations, stars with and without trapped modes and the "wiggles" in the sequences (see Fig. 4 of Baran 2012) may be used to deduce layer thicknesses and evolutionary ages. 
Frequency multiplets also provide useful information about rotation. From the multiplets detected so far (and lack of them in KIC 8302197, Baran et al. 2015, in press), we have found long rotation periods, from 25 to 100 days for apparently single stars. Even binaries with short periods down to 0.5 days rotate subsynchronously with periods longer than 7 days (see Table 2). Nearly all of the Kepler targets are dominated by $g$-mode pulsations yet ground-based observations have concentrated on $p$-mode pulsators. These are complimentary approaches except that ground-based observations tend to be short in duration. As such, frequency multiplets have only been observed in two sdB stars using ground-based observations (Reed et al. 2004, Baran et al. 2008). In order to apply seismology tools to more $p$-mode pulsators, we will need extended observations to resolve multiplets.

According to pulsation models, $p$ modes sample the outer atmosphere while $g$ modes sample much deeper, including a sensitivity to the core structure (Charpinet et al. 2014). As such, rotation profiles can be determined if multiplets of both types exist. As of this writing, only two stars have sufficient multiplets in both the $p$ - and $g$-mode regions to examine rotation. For KIC 10139564 the $p$ - and $g$-mode multiplets indicate the same rotation period (Baran et al. 2012) whereas multiplets in KIC 3527751 indicate the interior has a rotation period nearly three times longer than the envelope (Foster et al. 2015). We can hope that as we examine full Kepler data sets of the remaining pulsators, and as K2 observes more pulsators, that we can find more stars with simultaneous $p-$ and $g$-mode multiplets to examine rotation profiles.

To date, seven Kepler-observed sdb pulsators have been found to be binaries with periods from about 10 hours to 10 days (see Table 2 ). All of these binaries rotate subsynchronously. Pablo et al. (2012) examined two cases, both sdB+dM binaries, and placed some limits on companion mass and age. There are also four known sdB+WD binaries, with presumably $\sim 0.6 M_{\odot}$ companions, and the sdB stars still rotate subsynchronously and it is unlikely they are all young systems. Yet the only non-Kepler (ground-based) sdB pulsator that is both in a binary and has multiplets (Feige 48) is tidally locked at a period near 10 hours. These systems are all short period, and so post-common envelope binaries. As such, they can tell us a lot about angular momentum transfer with mass exchange/loss.

A more subtle impact is indicated in sFTs; amplitudes are varying significantly, often coming and going. This is not news (Kilkenny 2010) but sFTs now allow us the tools to examine how the amplitudes are changing for stars of differing temperatures, gravities, and presumably evolutionary stages. The sFTs in Fig. 3 are all from the same star yet show variety. They also indicate the importance of long-term observations. The rightmost panel is an excellent example of how when the star is observed will change how the pulsations are interpreted as this multiplet begins as a singlet and after two years appears as a quintuplet and at the end the central frequency no longer even has the highest amplitude.

From the 14 Kepler-observed sdB pulsators, there are $\sim 1150$ observationally identified so far. 775 of these are in frequency multiplets including multiplets of $\ell \geqslant 3$ and 850 were identified from period spacings. Thanks to Kepler data, we now have observationally constrained pulsations in sdB stars which can be used to constrain structure models. Yet even a thorough analysis of Kepler data will not provide a complete picture. In particular, there is a dearth of $p$-mode pulsators in the Kepler set, which will have to be filled in with ground-based data. 


\section{References}

Aerts, C., Christensen-Dalsgaard, J., \& Kurtz, D. W. 2010, Asteroseismology (1st ed. New York, Springer Astronomy \& Astrophysics Library)

Baran, A. S., Pigulski, A., \& O'Toole, S. J. 2008, MNRAS, 385, 255

Baran, A. S. \& Winans, A. J. 2012, AcA, 62, 343

Baran, A. S., et al. 2012, MNRAS, 424, 2686

Baran, A. S., Telting, J. H., Nemeth, P., Bachulski, Sz., \& Krzesinski, J. 2015a, A\& A, 573, 52

Baran, A. S., Telting, J. H., Nemeth, P., Østensen, R. H., Reed, M. D., \& Kiaeerad, F. 2015b, $A \& A$, in press

Charpinet, S., FontaineA G., Brassard, P., \& Dorman, B. 2002, ApJS, 139, 487

Charpinet, S., Fontaine, G., Brassard, P., \& Green, E. M. 2011, Nature, 480, 496

Charpinet, S., Brassard, P., Van Grootel, V., \& Fontaine, G. 2014 ASP-CS, 481, 179

Foster, H. M., Reed, M. D., Telting, J. H., Østensen, R. H., \& Baran, A. S 2015 ApJ, 805, 94

Heber, U. 1984, A\& $A, 130,119$

Heber, U. 2009, ARAA, 47, 211

Hu Haili, Nelemans, G., Aerts, C., \& Dupret, M.-A. 2009, A\&\&A, 508, 869

Jacobsen, E., \& Lyons, R. 2003, IEEE, SPeL, 74

Jenkins, J. et al. ApJL, 713, 87

Kilkenny, D. 2010, Ap\&SS, 329, 175

Ledeoux, P. 1951, ApJ, 114, 373

Miller Bertolami, M. M., Có rsico A. H., \& Althaus, L. G. 2012, ASP-CS, 452, 175

Moehler, S., Heber, U., Lemke, M., \& Napiwotski, R. 1998, A\&A, 339, 537

Østensen, R.H. 2010 AN, 331, 1026

Østensen, R. H., Telting, J. H., Reed, M. D., Baran, A. S., Nemeth, P., \& Kiaeerad, F. 2014 $A \mathscr{E} A, 569,15$

Pablo Herbert, et al. 2012, MNRAS, 422, 1343

Paxton, B., Bildsten, L., Dotter, A., Herwig, F., Lesaffre, P., \& Timmes, F. 2011, ApJS, 192, 3

Reed, M. D. et al. (The Whole Earth Telescope Collaboration) 2004, MNRAS, 348, 1164

Reed, M. D., et al. 2011, MNRAS, 414, 2885

Reed, M. D., Foster, H., Telting, J. H., Østensen, R. H., Farris, L. H., Oreiro, R., \& Baran, A. S. 2014 MNRAS, 440, 3809

Saffer, R. A., Bergeron, P., Koester, D., \& Liebert, J. 1994 ApJ, 432, 351

Unno, W., Osaki, Y., Ando, H., \& Shibahashi, H. 1979, Nonradial Oscillations of Stars (Tokyo: University of Tokyo Press) bibitem[Van Grootel et al. 2013]vangrootel13 Van Grootel, V., Charpinet, S., Brassard, P., Fontaine, G., Green, E. M. 2013 A $\& A$, 553, 97 